\title{
The first composite score predicting Digital Ulcers in systemic sclerosis patients using Clinical data, Imaging and Patient history-CIP-DUS
}

\author{
S. Friedrich ${ }^{1,2+}$, S. Lüders ${ }^{1,3 \dagger}$, J. Klotsche ${ }^{4,5}$, G. R. Burmester ${ }^{1}$, G. Riemekasten ${ }^{6}$ and S. Ohrndorf ${ }^{*^{*}}$
}

\begin{abstract}
Background: Digital ulcers (DU) present a challenging complication in systemic sclerosis (SSc). The aim of this study was to combine clinical characteristics and imaging methods to a composite score for the prediction of DU in SSc patients.

Methods: Seventy-nine SSc patients received clinical examination, their patient history was taken and nailfold capillaroscopy (NC), colour Doppler ultrasonography (CDUS) and fluorescence optical imaging (FOI) of the hands were performed at baseline. Newly developed DU over a period of approximately 12 months were registered. We used criteria with area under the curve (AUC) of at least 0.6 in regard to the development of these new DU to create the score (CIP-DUS, clinical features, imaging, patient history—digital ulcer score).

Results: Twenty-nine percent of all SSc patients developed new DU during follow-up (48.1\% diffuse, 18.4\% limited SSc). Based on the cross-validated (CV) AUC, a weight (cVAUC > 0.6 and $\leq 0.65: 1$; cVAUC $>0.65$ and $\leq 0.7: 2$; cVAUC $>0.7$ : 3) was assigned to each selected parameter. The performance of the final CIP-DUS was assessed with and without the CDUS/FOI component. For the scleroderma patterns in NC, three points were appointed to late, two to active and one point to early capillaroscopy pattern according to Cutolo et al. The CIP-DUS including the CDUS and FOI parameters resulted in a good diagnostic performance (AUC after cross-validation: $0.83,95 \% \mathrm{Cl} 0.74$ to 0.92 ) and was well calibrated (chi-square $=12.3, p=0.58$ ). The cut-off associated with the maximum of sensitivity and specificity was estimated at $\geq 10$ points resulting in a sensitivity of $100 \%$ and specificity of $74 \%$ for new DU during follow-up. Excluding CDUS and FOI parameters leads to a non-statistically significant lower performance (AUC after cross-validation: $0.81,95 \% \mathrm{Cl} 0.72$ to 0.91). However, including CDUS and FOI resulted in a better classification of patients in respect to the outcome new DU in follow-up due to significantly better reclassification performance $(\mathrm{NRI}=62.1, p=0.001)$ and discrimination improvement $(\mathrm{IDI}=9.7, p=0.01)$.

(Continued on next page)
\end{abstract}

\footnotetext{
* Correspondence: sarah.ohrndorf@charite.de

${ }^{\dagger} \mathrm{S}$. Friedrich and S. Lüders contributed equally to this work.

'Department of Rheumatology and Clinical Immunology, Charité Universitätsmedizin Berlin, Berlin, Germany

Full list of author information is available at the end of the article
}

C C The Author(s). 2020 Open Access This article is licensed under a Creative Commons Attribution 4.0 International License, which permits use, sharing, adaptation, distribution and reproduction in any medium or format, as long as you give appropriate credit to the original author(s) and the source, provide a link to the Creative Commons licence, and indicate if changes were made. The images or other third party material in this article are included in the article's Creative Commons licence, unless indicated otherwise in a credit line to the material. If material is not included in the article's Creative Commons licence and your intended use is not permitted by statutory regulation or exceeds the permitted use, you will need to obtain permission directly from the copyright holder. To view a copy of this licence, visit http://creativecommons.org/licenses/by/4.0/ The Creative Commons Public Domain Dedication waiver (http://creativecommons.org/publicdomain/zero/1.0/) applies to the data made available in this article, unless otherwise stated in a credit line to the data. 
(Continued from previous page)

Conclusion: A new score was introduced with the aim to predict digital ulcers. If applied correctly and with the new imaging techniques proposed, all patients at risk of digital ulcers throughout 12 months could be identified.

Keywords: Systemic sclerosis, Capillaroscopy, Colour Doppler ultrasound, Fluorescence optical imaging, Modified Rodnan skin score, Disturbed microcirculation, Digital ulcers

\section{Background}

Digital ulcers (DU) affect more than a third of patients with systemic sclerosis (SSc) over time ensuing a considerable decrease in quality of life [1]. Different risk factors for the development of DU have already been described: male gender, history of digital ulcers, pathologic CSURI (Capillaroscopy Skin Ulcers Risk Index), and altered ESR (erythrocyte sedimentation rate) [2]. So far, limited efforts have been made to create a scoring system using capillaroscopic findings. First, CSURI was developed, which identifies patients at risk of developing new DU in a 3-month follow-up [3-5]. However, a multicentre study showed that $40 \%$ of their patients were not assessable with CSURI due to the absence of megacapillaries, which are an essential part of the index's equation [6]. Furthermore, Caramaschi et al. developed a score of risk factors that was associated with ischemic digital ulcers in SSc patients undergoing Iloprost treatment. They identified four variables with high ORs for ischemic DU: age at disease onset $<47.0$ years (OR 6.17), delay in beginning Iloprost therapy $>18$ months (OR 5.70), history of smoking (OR 6.80), and presence of contractures (OR 6.50). Thus, they designed an additive model with one point per risk factor present. ROC analysis showed promising results with an AUC of 0.836 (95\% CI 0.7360.937) [7]. Manfredi et al. proposed a composite predictive model including "capillaroscopic, demographic and clinic-serological parameters" to identify patients at risk for new digital ulcers [2].

In other rheumatologic diseases, the use of composite scores is performed as a standard procedure, either to assess disease activity or severity, e.g. SLAM (Systemic Lupus Activity Measure), SLEDAI (Systemic Lupus Erythematosus Disease Activity Index) or BILAG (British Isles Lupus Assessment Group) for systemic lupus erythematosus; and DAS28 (Disease activity score 28), SDAI (simple disease activity index), and CDAI (clinical disease activity index) for rheumatoid arthritis, and many more in other indications such as psoriatic arthritis or ankylosing spondylitis. Most of these composite scores have been developed to reflect current disease activity, but also in order to account the emergence of new clinical manifestations of the underlying disease. Composite scores in the field of rheumatology usually take into account biomarkers or laboratory markers as well as clinical findings and information given by patients.
So far, there has not yet been presented a composite score created for patients with systemic sclerosis. Our aim was to create a composite score for the prediction of digital ulcers in SSc patients combining clinical data, imaging, and patient history based on the results that were recently published by our group [8].

\section{Patients and methods}

Seventy-nine in- and out-patients with SSc were recruited. A signed informed consent was obtained from all patients. Three patients dropped out during the follow-up period: one patient withdrew her consent; two patients unfortunately died. After a medium of 12 months, we asked for newly developed digital ulcers during the follow-up period.

The included SSc patients received clinical examination, nailfold capillaroscopy (NC), colour Doppler ultrasonography (CDUS) [8, 9], and fluorescence optical imaging (FOI) $[8,10]$ of both hands at baseline, and also their patient history was taken as described in detail in our previous publications [8-10]. The two examiners were not blinded to patient history or clinical manifestations of the disease when performing the imaging techniques. Furthermore, NC was performed to assess for capillary density and capillaroscopy patterns (late, active, and early) introduced by Cutolo et al. in the year 2000.

\section{Statistical analyses}

The association of each potential score component with the likelihood of newly developed DU was estimated by means of a logistic regression model. The area under curve (AUC) was determined to assess the predictive performance of each predictor variable. $K$-fold $(k=10)$ cross-validation was used to evaluate the predictive performance of each parameter to generate a more realistic estimate of predictive performance [11]. The idea of cross-validation is to randomly divide the data into $k$ equal-sized parts. The $k$ th part is left out, the model is fit to the other $k-1$ parts, and then predictions for the left-out $k$ th part are obtained. A parameter was included into the score if the resulting AUC reached a value of at least 0.6. Based on the cross-validated (cv) AUC, a weight (cvAUC $>0.6$ and $\leq 0.65: 1$; cvAUC $>0.65$ and $\leq$ 0.7: 2; cvAUC >0.7: 3) was assigned to each selected parameter. The performance of the final CIP-DUS was assessed with and without the CDUS/FOI component. 
The improved model performance by adding the CDUS/ FOI component was evaluated by comparing the AUC using the algorithm suggested by DeLong [12]. In addition, the net reclassification improvement (NRI) and integrated discrimination improvement (IDI) [13] were calculated to evaluate the appropriate or inappropriate reclassification of patients after adding CDUS/FOI. Finally, the calibration of the logistic model was tested by calculating Hosmer-Lemeshow chi-square test. Statistical analyses were performed with STATA 12.1 (StataCorp. 2011. Stata Statistical Software: Release 12. College Station, TX: StataCorp LP).

\section{Results}

Of the 76 SSc patients (62 female patients, $n=43$ with limited cutaneous SSc and $n=19$ with diffuse cutaneous SSC) that were questioned at 12 months follow-up, 29\% reported newly developed DU (48.1\% of patients with diffuse SSc and $18.4 \%$ of patients with limited SSc) (see Supplementary Table and [8]). The obtained AUC were calculated for potential parameters to be included in the CIP-DUS after $k$-fold cross-validation. A strong association with newly developed DU during follow-up was observed for history of digital ulcer and/or pitting scars (OR 36.2; 95\% CI 2.1 to 626.9; AUC 0.77), present digital ulcers and/or pitting scars at baseline (OR 15.7; 95\%CI 3.3 to 74.3; AUC 0.76), and the NC pattern (OR 18.6; 95\%CI 1.1 to 326.4; AUC 0.70). The modified Rodnan skin score was moderately associated with the likelihood for newly developed ulcers at follow-up. A weight of one was assigned to the SSc subtype, pulmonary arterial hypertension, reduced capillary density $(n<7 / \mathrm{mm})$ in digit III of the right hand in $\mathrm{NC}$, missing initial enhancement in FOI in digit III of the right hand, and percentage of pathologic (i.e. narrowed or occluded) vessels $>35 \%$ in CDUS based on the AUC. For the scleroderma patterns in nailfold capillaroscopy as introduced by Cutolo et al., three points were appointed to late, two to active, and one to early capillaroscopy pattern (see Table 1).

The CIP-DUS including the CDUS and FOI parameters resulted in a good diagnostic performance (AUC after cross-validation: $0.83,95 \% \mathrm{CI} 0.74$ to 0.92 , see Fig. 1 ) and was well calibrated (chi-square $=12.3, p=0.58$ ). The cut-off associated with the maximum of sensitivity and specificity was estimated at $\geq 10$ points resulting in a sensitivity of $100 \%$ and specificity of $74 \%$ for new DU during follow-up.

Alternatively, the score was calculated without inclusion of the CDUS and FOI parameters. It resulted in a slightly lower diagnostic performance (AUC after crossvalidation: $0.81,95 \% \mathrm{CI} 0.72$ to 0.91 ) as compared to CIP-DUS. The difference was not statistically significant (chi-square $=1.6, p=0.20$, Fig. 1). But the inclusion of CDUS and FOI resulted in a significantly better reclassification performance $(\mathrm{NRI}=62.1, \quad p=0.001)$ and discrimination improvement (IDI $=9.7, p=0.01$ ) in comparison to the score in absence of CDUS and FOI indicating a better classification of patients in respect to the outcome DU at follow-up. In more detail, 14 (64\% of 22 patients with DU at follow-up) had a higher predicted probability for a positive outcome by including CDUS and FOI, whereas 18 patients (33\% of 54 patients without DU at follow-up) had a lower predicted probability for a positive outcome. The other way around, only 4 patients $(18 \%)$ were assigned to a lower risk and 9 patients (18\%) to a higher risk in patients with and without a DU at follow-up, respectively. It means that CIP-DUS (including CDUS and FOI) tended to result in higher predicted probabilities in patients with DU at follow-up and lower predicted probabilities in patients without, indicating a better diagnostic performance (sensitivity and specificity).

\section{Discussion}

The aim of our project was to combine patient history, clinical and imaging findings in order to create a composite score able to determine the individual risk for the development of new DU in SSc patients during a 12month follow-up. The odds ratios of known risk factors and other features were obtained.

Consistent with previous literature, current or previous digital ulcers and/or pitting scars were important factors in the recurrence of this complication [2,14]. Our data also suggest a link between skin involvement (represented by a diffuse cutaneous subtype or a high mRSS) and the DU development [8]. Sunderkötter et al. [14] also found that a diffuse skin sclerosis significantly impacted on the appearance of DU in multivariate analysis, but only when pulmonary arterial hypertension was present. Here, in univariate analysis, both the diffuse subtype and pulmonary arterial hypertension (PAH) were associated with digital ulcers in a 12-month follow-up.

While PAH as a sign of systemic vasculopathy already links to the pathophysiology of digital ulcers, the evidence of micro- and macrovasculopathy in imaging is an established tool in the diagnostic and risk stratification of SSc patients. In accordance with our results, Smith et al. [15] found a more severe capillaroscopic pattern predictive for future severe peripheral vascular involvement. In the CAP study, the mean number of capillaries per millimetre in the middle finger of the dominant hand was identified as one of the strongest risk factors for new DU [16]. This is comparable with our finding that a reduced capillary density in the right middle finger indicated a higher risk of ulcers. Interestingly, pathologic findings in this finger detected by FOI and CDUS (not significant) were also associated with our primary end point. In CDUS however, an overall high percentage of pathologic vessels showed better predictive values. 


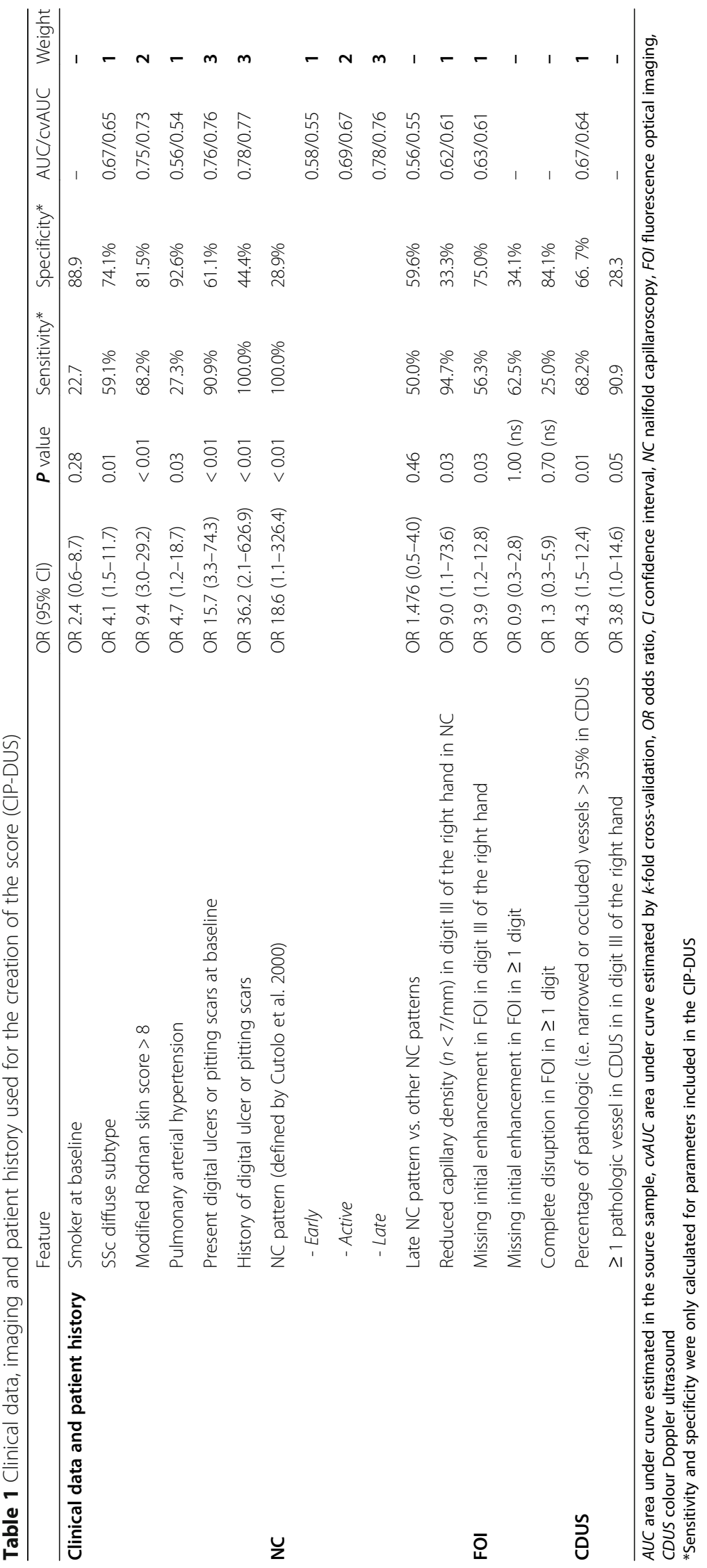



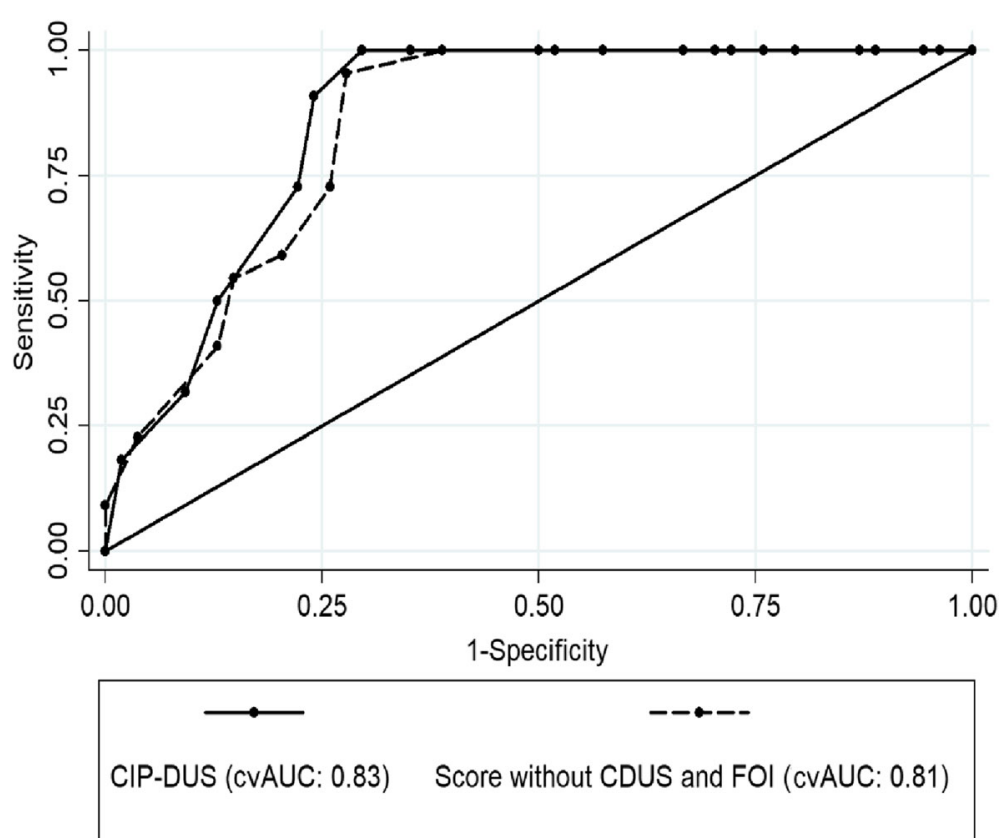

Fig. 1 Receiver operating characteristic (ROC) of the CIP-DUS and the score in absence of CDUS and FOI regarding the development of new digital ulcers in a 12-month follow-up

While the single identified risk factors showed either low sensitivity (skin involvement, CDUS, FOI) or low specificity (DU presence/history, capillaroscopy) levels (data not shown), we aimed to combine the factors to a predictive score. The final composite score proved good diagnostic performance with perfect sensitivity of $100 \%$ and good specificity of $74 \%$ at a cut-off of $\geq 10$ points. Excluding the uncommon imaging techniques CDUS and FOI, the score showed a slightly lower diagnostic performance in comparison to the complete CIP-DUS, which was not statistically significant. However, reclassification performance and discrimination improvement were significantly better when the full CIP-DUS (including CDUS and FOI) was assessed.

One limitation of the proposed CIP-DUS is the limited availability of the suggested imaging methods CDUS and FOI. Nevertheless, our data revealed an only slight decrease of the score's performance when excluding those two imaging methods. This shows that with the commonly obtained information (clinical data, NC, patient history), which every clinician treating SSc patients should possess, a practical and powerful score assessment of the risk to develop new DU is still possible and should be integrated in clinical routine.

The assessment of DU is a challenging task. Occurrence of DU during follow-up was assessed via a telephone or personal interview and patients were instructed (using pictures) at baseline to look out for and document newly formed ulcers on their hands in order to improve accuracy at follow-up. A limitation of this study is the insufficient differentiation made between ischemic and traumatic ulcers.

Advantages of this study include the relatively long follow-up period of 12 months and the thorough characterisation of the patients including demographic, clinical, laboratory, and imaging data. Due to the complex pathophysiology of systemic sclerosis and its complications such as DU, taking all these factors into account is paramount when trying to reduce the risk of DU in the future.

As this is a pilot project, the predictive values of the composite score have unfortunately not yet been applied on an independent cohort. This should be conducted in future studies on a larger scale of patients-if only in the reduced form without FOI and CDUS. Even the reduced CIP-DUS, which integrates the usually present patient information about clinical presentation, patient history as well as NC pattern and capillary density can provide a powerful risk assessment for future DU in SSc patients.

\section{Conclusions}

The new composite score CIP-DUS predicts digital ulcer development throughout a 12-month follow-up and identifies all "at risk" patients. Even without the new imaging features, which might not be available to all clinicians, the score still shows good performance in regard to detecting patients at risk for new DU development. 


\section{Supplementary information}

Supplementary information accompanies this paper at https://doi.org/10. 1186/s13075-020-02235-7.

Additional file 1:. Supplement Table. Baseline patient characteristics, including diagnosis, sex, age $( \pm S D)$, Raynaud's phenomenon, digital ulcers and nailfold capillaroscopy patterns as described by Cutolo et al. (table cited from [8])

\section{Abbreviations}

95\%Cl: 95\% confidence interval; ACR/EUSTAR: The American College of Rheumatology/European League Against Rheumatism; AUC: Area under the curve; cvAUC: Cross-validated area under the curve; BILAG: British Isles Lupus Assessment Group; CDA: Clinical disease activity index; CDUS: Colour Doppler ultrasonography; CIP-DUS: Clinical data, imaging, patient history-digital ulcer score; CSURI: Capillaroscopy Skin Ulcers Risk Index; DAS28: Disease activity score 28; DU: Digital ulcers; FOl: Fluorescence optical imaging; ESR: Erythrocyte sedimentation rate; ICG: Indocyanine green; mRSS: Modified Rodnan skin score; OR: Odds ratio; PAH: Pulmonary arterial hypertension; ROC: Receiver operating characteristic; RP: Raynaud's phenomenon; SSc: Systemic sclerosis; SDAl: Simple disease activity index; SLAM: Systemic Lupus Activity Measure; SLEDAl: Systemic Lupus Erythematosus Disease Activity Index

\section{Acknowledgements}

We thank Prof. Wolfgang A. Schmidt for his help in training the CDUS examiners (SF, SL).

\section{Authors' contributions}

All authors participated in the preparation of the manuscript. SF contributed to the study design, data acquisition and analysis, and drafting of the manuscript. SL contributed to the study design, data acquisition and analysis, and drafting of the manuscript. JK contributed to the data analysis and drafting the manuscript revision. GR contributed to the study design and manuscript revision. SO contributed to the study design and manuscript revision. All authors read and approved the final version of the manuscript.

\section{Funding}

This work was supported by the German Federal Ministry of Education and Research (BMBF) (ArthroMark project, Subproject No. 7), the Actelion project "INDIZ", and the Miriam Lichy Foundation.

\section{Availability of data and materials}

The datasets used and/or analysed during the current study are available from the corresponding author on reasonable request.

\section{Ethics approval and consent to participate}

The local ethics committee of the Charité - Universitätsmedizin Berlin, granted ethical approval (reference no. EA1/269/13). Signed informed consent to participate was obtained from all patients.

\section{Consent for publication}

Not applicable.

\section{Competing interests}

The authors declare that they have no competing interests.

\section{Author details}

'Department of Rheumatology and Clinical Immunology, Charité Universitätsmedizin Berlin, Berlin, Germany. ${ }^{2}$ Department of Radiology, Charité - Universitätsmedizin Berlin, Berlin, Germany. ${ }^{3}$ Department of Gastroenterology and Rheumatology, Charité - Universitätsmedizin Berlin, Berlin, Germany. ${ }^{4}$ German Rheumatism Research Center, a Leibniz Institute, Berlin, Germany. ${ }^{5}$ Institute for Social Medicine, Epidemiology and Health Economics, Charité - Universitätsmedizin Berlin, Berlin, Germany.

${ }^{6}$ Department of Rheumatology and Clinical Immunology, University of Schleswig-Holstein, Lübeck, Germany.
Received: 5 November 2019 Accepted: 3 June 2020

Published online: 15 June 2020

\section{References}

1. Botzoris $V$, Drosos $A A$. Management of Raynaud's phenomenon and digital ulcers in systemic sclerosis. Joint Bone Spine. 2011;78(4):341-6.

2. Manfredi A, Sebastiani M, Carraro V, ludici M, Bocci M, Vukatana G, et al. Prediction risk chart for scleroderma digital ulcers: a composite predictive model based on capillaroscopic, demographic and clinico-serological parameters. Clin Hemorheol Microcirc. 2015;59(2):133-43.

3. Sebastiani M, Manfredi A, Colaci M, D'amico R, Malagoli V, Giuggioli D, et al. Capillaroscopic skin ulcer risk index: a new prognostic tool for digital skin ulcer development in systemic sclerosis patients. Arthritis Rheum. 2009;61: 688-94.

4. Sebastiani M, Manfredi A, Vukatana G, Moscatelli S, Riato L, Bocci M, et al. Predictive role of capillaroscopic skin ulcer risk index in systemic sclerosis: a multicentre validation study. Ann Rheum Dis. 2012;71:67-70.

5. Smith V, DeKeyser F, Pizzorni C, Van Praet JT, Decuman S, Sulli A, et al. Nailfold capillaroscopy for day-to-day clinical use: construction of a simple scoring modality as a clinical prognostic index for digital trophic lesions. Ann Rheum Dis. 2011;70:180-3

6. Walker UA, Jaeger VK, Bruppacher KM, Dobrota R, Arlettaz L, Banyai M, et al. Prospective evaluation of the capillaroscopic skin ulcer risk index in systemic sclerosis patients in clinical practice: a longitudinal, multicentre study. Arthritis Res Ther. 2018;20(1):239.

7. Caramaschi P, Martinelli N, Volpe A, Pieropan S, Tinazzi I, Patuzzo G, et al. A score of risk factors associated with ischemic digital ulcers in patients affected by systemic sclerosis treated with iloprost. Clin Rheumatol. 2009;28: 807-13.

8. Friedrich S, Lüders S, Glimm AM, Werner SG, Schmittat G, Burmester GR, et al. Association between baseline clinical and imaging findings and the development of digital ulcers in patients with systemic sclerosis. Arthritis Res Ther. 2019:21(1):96.

9. Lüders S, Friedrich S, Ohrndorf S, Glimm AM, Burmester GR, Riemekasten G, et al. Detection of severe digital vasculopathy in systemic sclerosis by colour Doppler sonography is associated with digital ulcers. Rheumatology (Oxford). 2017:56(11):1865-73.

10. Friedrich S, Lüders S, Werner SG, Glimm AM, Burmester GR, Riemekasten G, et al. Disturbed microcirculation in the hands of patients with systemic sclerosis detected by fluorescence optical imaging: a pilot study. Arthritis Res Ther. 2017;19(1):87.

11. LeDell $\mathrm{E}$, Petersen $\mathrm{M}$, van der Laan M. Computationally efficient confidence intervals for cross-validated area under the ROC curve estimates. Electron J Stat. 2015;9(1):1583-607.

12. DeLong ER, DeLong DM, Clarke-Pearson DL. Comparing the areas under two or more correlated receiver operating characteristic curves: a nonparametric approach. Biometrics. 1988;44:837-45.

13. Pencina MJ, D'Agostino RB Sr, D'Agostino RB Jr, Vasan RS. Evaluating the added predictive ability of a new marker: from area under the ROC curve to reclassification and beyond. Stat Med. 2008;27(2):157-72.

14. Sunderkötter C, Herrgott I, Brückner C, Moinzadeh P, Pfeiffer C, Gerß J, et al. Comparison of patients with and without digital ulcers in systemic sclerosis: detection of possible risk factors. Br J Dermatol. 2009;160(4):835-43.

15. Smith V, Decuman S, Sulli A, Bonroy C, Piettte Y, Deschepper E, et al. Do worsening scleroderma capillaroscopic patterns predict future severe organ involvement? A pilot study. Ann Rheum Dis. 2012;71(10):1636-9.

16. Cutolo M, Herrick AL, Distler O, Becker MO, Beltran E, Carpentier $P$, et al. Nailfold videocapillaroscopic features and other clinical risk factors for digital ulcers in systemic sclerosis: a multicenter, prospective cohort study. Arthritis Rheumatol. 2016;68(10):2527-39.

\section{Publisher's Note}

Springer Nature remains neutral with regard to jurisdictional claims in published maps and institutional affiliations. 